\title{
Study of Serum Homocysteine Level in Young Patients with Myocardial Infarction and Cerebrovascular Stroke
}

\author{
Meena Rajiv Daveshwar ${ }^{1}$, Hiral Samir Shah ${ }^{2 *}$ and Ankit Zaveri ${ }^{2}$ \\ 'Pathology Department, SSG Hospital and Medical College Baroda. INDIA \\ ${ }^{2}$ Pathology Department, SSG Hospital and Medical College Baroda.
}

\begin{abstract}
Background: Nowadays, vascular disease is the leading cause of death and disability. India too is facing epidemic of cardiovascular and cerebrovascular disease. The established risk factors for heart disease and stroke account for one half to three fourth of cases. The remainders are the result of other factors that promote atherosclerosis. Elevated blood levels of homocysteine have been linked to increased risk of premature coronary artery disease, stroke and thromboembolism, even among people who have normal cholesterol levels. Hence screen for homocysteine is recommended in patients with premature atherosclerotic disease and unexplained venous thrombosis.

Methods: The present study was performed to correlate the levels of serum homocysteine levels in young patients $(</=40$ yrs $)$ with myocardial infarction and ischemic cerebrovascular stroke. Thirty cases were studied along with assessment of their risk factor.

Result: It was observed that serum homocysteine levels were significantly raised in coronary artery disease and ischemic stroke patients.

Conclusion: Increase levels of homocysteine is an important factor that assesses risk independently for coronary artery disease and ishchemic stroke.
\end{abstract}

\section{Keywords: Homocysteine, Myocardial Infarction, Ischemic Stroke, Coronary Artery Disease}

\section{Introduction}

Despite impressive stride and management over past three decades, acute Myocardial Infarction and stroke contributes to a major public health problem in the industrialized world and becoming an important health problem in developing countries. The risk factors are both modifiable and nonmodifiable. American heart association (AHA) had given guidelines for risk intervention and primary prevention of cardiovascular disease and ischemic stroke. ${ }^{[1]}$

During past few years, elevated serum homocysteine concentration is emerging as a risk factor for premature coronary artery disease, stroke and thromboembolism. The prevalence rate of coronary artery disease (CAD) in our country is $80-120 / 1000$ population. ${ }^{[2]}$ The present study was performed with an aim to evaluate homocysteine as an independent risk factor for Myocardial Infarction (MI) and Ischemic stroke.

\section{Materials and Methods}

The study of homocysteine levels in young patients $(</=40$ yrs) with MI and ischemic stroke was carried out at SSG Hospital, Medical College, Baroda over period of one and half year. The study population consisted of two separate groups of 30 young patients having MI and ischemic stroke. Each diagnosed by clinical signs, symptoms and other supportive investigations. Two separate control groups were also included of same age group without Ischemic heart disease (IHD) and cerebrovascular (CV) stroke.

The inclusion criteria of MI patients in the study are-(i) Chest pain $>30$ minutes, not responding of sublingual nitroglycerin tab. (ii) presence of pathological Q wave along with ST segment elevation and $\mathrm{T}$ wave inversion. (iii)significant rise in CK MB isoenzyme on $1^{\text {st }}$ and $2^{\text {nd }}$ day, While for ischemic stroke, they are (i)clinical signs and symptoms of weakness, vomiting, giddiness, altered sensorium, visual disturbances, speech disturbances, asymmetry of face (ii) CT scan finding of ischemic stroke.

Each patients and control group individual had been evaluated clinically as well as using standard protocol, which included hemogram, urine examination, FBS and PP2BS, Blood urea, S. creatinine, lipid profile and serum homocysteine level. The patients were also evaluated for risk factors (Hypertension, Hyperlipidemia, smoking status, obesity, diabetes mellitus, alcohol intake and family history).

The serum homocysteine levels were measured 8-12 weeks after attack of MI and CV stroke. It is measured in a 10hour fasting blood sample in both case and control groups. Rapid processing of sample done as homocysteine quickly leaches out of red blood cells. The S. homocysteine level was assayed using Immulite kit. Normal range being 5-15 micro $\mathrm{mol} / \mathrm{L}$. 


\section{Result}

In present study it was observed that incidence of MI and Cerebrovascular stroke were more in male population, with a male: female ratio being 9:1 and 14:1 respectively. Chest pain $(100 \%)$ and Ghabharaman $(63.3 \%)$ were the most common presenting symptom of Myocardial infarction, while weakness $(93.3 \%)$ was the most common symptom of CV stroke.
Lipid profile study revealed that LDL and serum triglyceride levels were higher in cases of myocardial infarction (Table 1). Biochemical assessment of fasting serum homocysteine levels strongly suggests that mean level was significantly higher in patients with Myocardial infarction and $\mathrm{CV}$ stroke as compared to control group (Fig 1). By risk factor assessment, we revealed that smoking $(73.33 \%)$ was the commonest risk factor associated in both the groups.

Table 1: Lipid profile and serum homocysteine level of study population.

\begin{tabular}{|c|c|c|c|c|}
\hline & \multicolumn{2}{|c|}{ Myocardial infarction } & \multicolumn{2}{|c|}{ Cerebrovascular stroke } \\
\hline & Cases & Control & Cases & Control \\
\hline Total cholesterol(mean) & 174.33 & 174.60 & 183.13 & 167.93 \\
\hline$<200 \mathrm{mg} \%$ (no.of patients) & 26 & 31.43 & 21 & 28.07 \\
\hline$\geq 200 \mathrm{mg} \%$ (no.of patients) & 4 & 19 & 9 & 28.3 \\
\hline HDL(mean) & 41.73 & 44.06 & 41.7 & 42.53 \\
\hline LDL(mean) & 106.14 & 97.53 & 103.72 & 101 \\
\hline S. Triglyceride(mean) & 156.2 & 149 & 149.23 & 150.26 \\
\hline Cholesterol/HDL ratio & 4.34 & 3.97 & 4.4 & 3.95 \\
\hline Serum homocysteine level (mean) & $29.77+/-6.97$ & $11+/-1.96$ & $28.14+/-7.88$ & $11.18+/-1.82$ \\
\hline
\end{tabular}

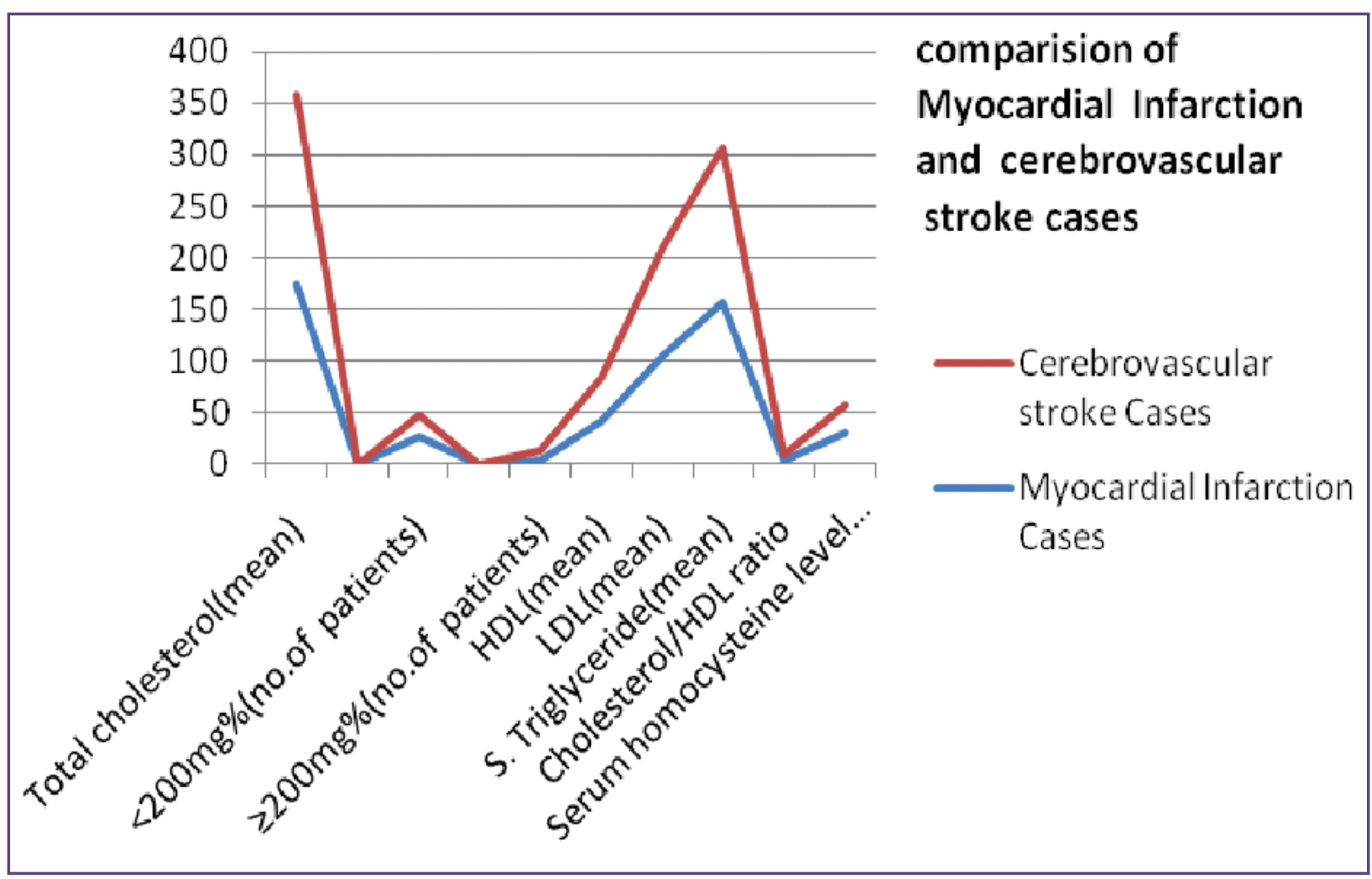

Fig. 1: comparison of Myocardial Infarction and Cerebrovascular stroke cases. 


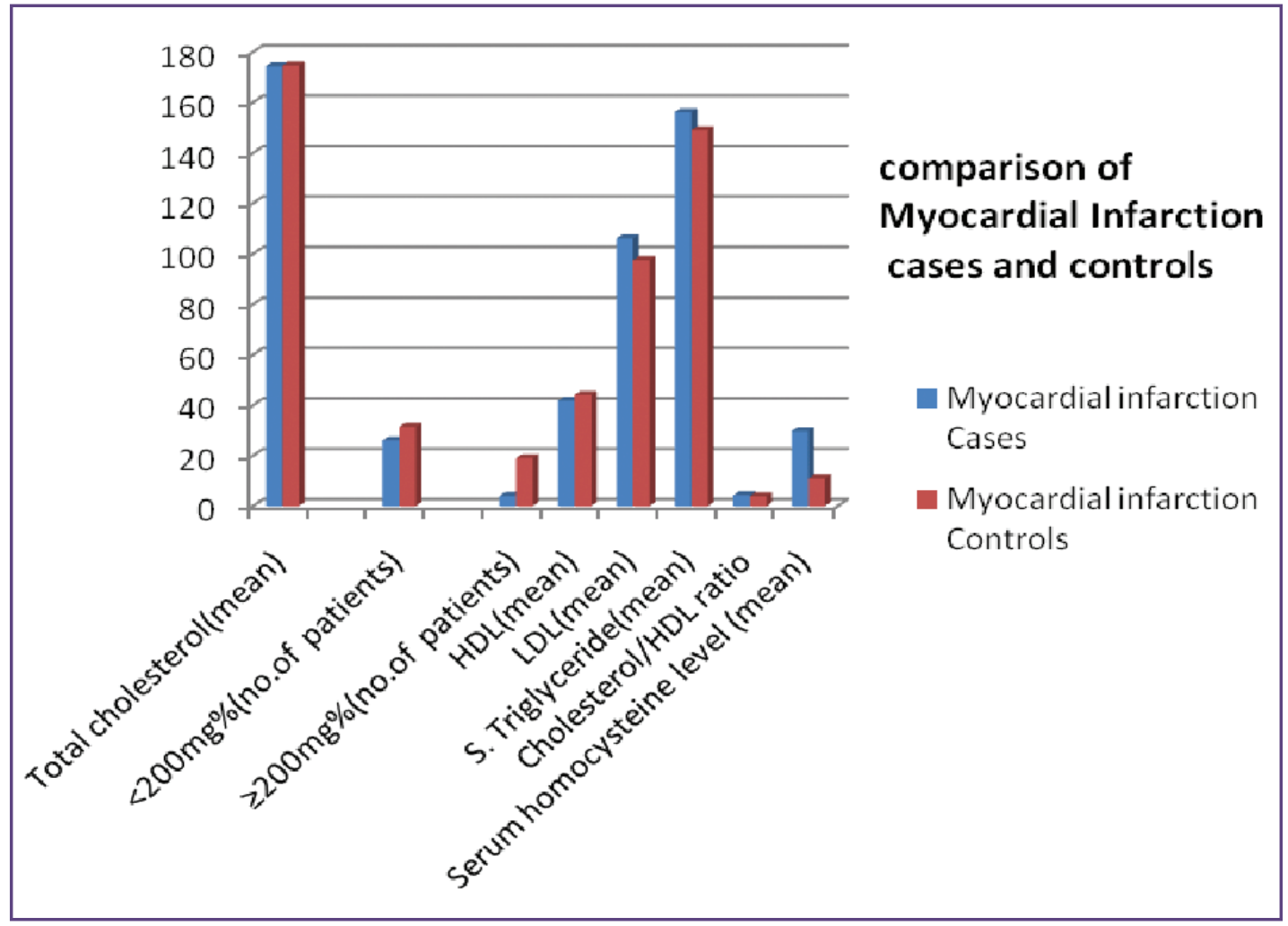

Fig. 2: illustrates comparison of Myocardial Infarction cases and controls.

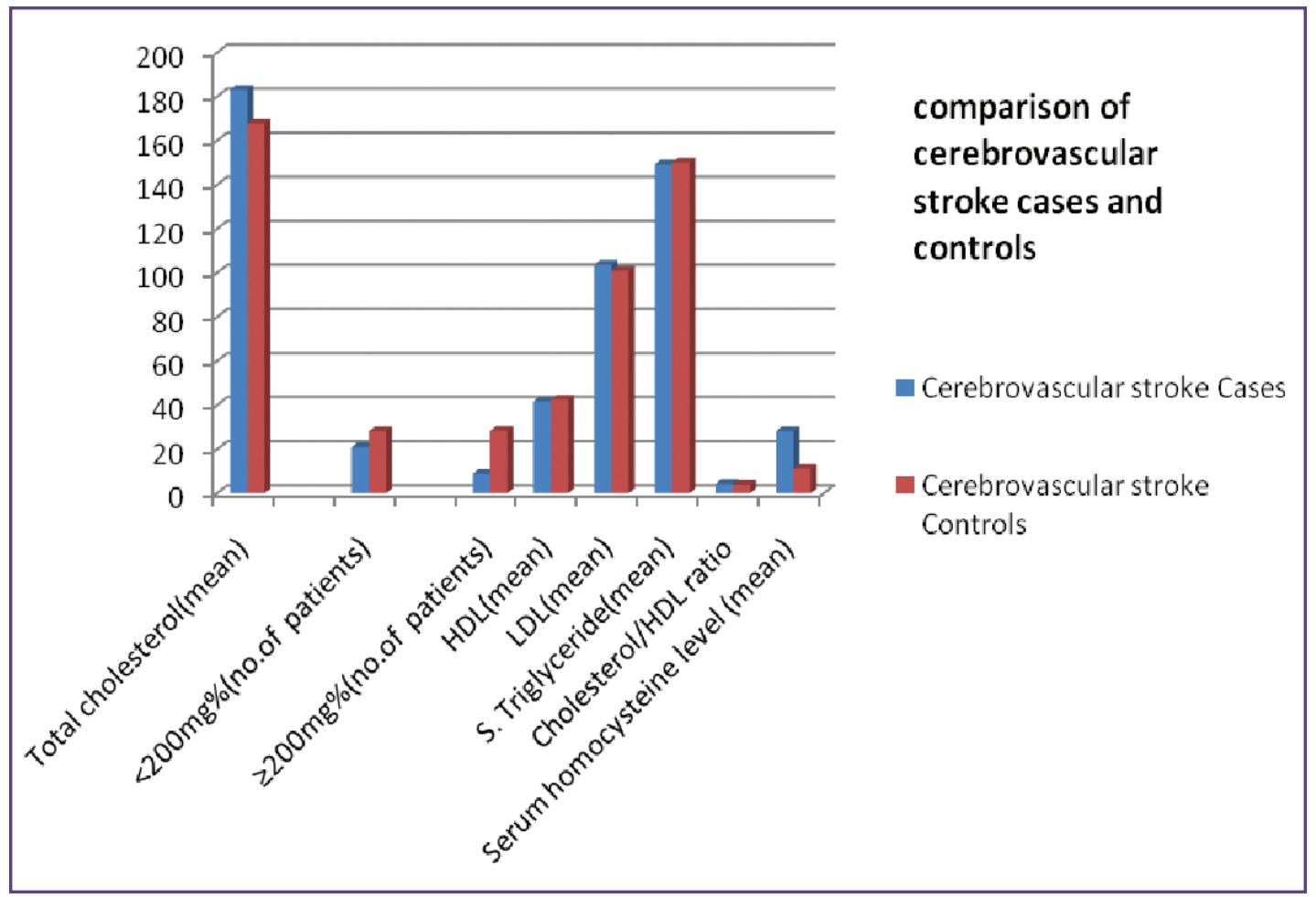

Fig. 3: illustrates comparison of cerebrovascular stroke cases and controls. 


\section{Discussion}

The word "homocysteine" was coined by du Vigneaud and coworkers, who discovered this compound approximately 60 years ago after isolation from a urinary bladder stone. Homocysteine metabolism is governed by several important B-vitamins such as folic acid, vitamin B12 and vitamin B6. It is found that elevated level of homocysteine in the blood may increases person's chance of developing heart disease, stroke or other blood flow disorders ${ }^{[3]}$

Sulphhydryl group of homocysteine is reactive and may undergo redox reaction and cause oxidative stress. Abnormal homocysteine levels contribute to atherosclerosis by three ways (1) Direct toxic effect damages the endothelial cells. (2) Interference with the clotting factor and (3) Oxidation of low-density lipoproteins (LDL) gets converted in to small atherogenic forms. ${ }^{[4]}$

The lipid profile study suggests that the difference in homocysteine levels observed between patients with serum cholesterol $>/=200 \mathrm{mg} \%$ and those patients with serum cholesterol $<200 \mathrm{mg} \%$ was statistically significant ( $p$ value $=0.0001$ ) suggesting that in patients who did not have high levels of serum cholesterol, the higher levels of serum homocysteine triggered the coronary artery disease. Thus homocysteine is an important and independent predictor of myocardial infarction however the difference in homocysteine levels observed was not significant $(p$ value $=0.9492)$ in cererovascular stroke in this study. HDL-C, triglycerides and total cholesterol /HDL-C ratio are important risk factors for myocardial infarction and cererovascular stroke. The low levels of LDL-C and high levels of HDL-C do not protect against the homocysteine induced myocardial infarction or cerebrovascular stroke. ${ }^{[5]}$

High serum Homocysteine levels appear to be clearly associated and increased risk of cardiovascular and cerebrovascular disease. It was linked to - MI, recurrent coronary events, premature coronary heart disease (CHD), total mortality or adverse outcomes after angioplasty. JAMA, 2002, reports of a meta analysis evaluated data from 30 prospective and retrospective studies involving 5073 Ishchemic Heart Disease and stroke patients. After adjustment for known cardiovascular risk factors, a $25 \%$ lower homocysteine concentration (about $3 \mathrm{micro} \mathrm{mol} / \mathrm{L}$ ) in prospective studies was associated with lower risk of IHD as compared to retrospective study. (odds ratio $0.89,95 \%$ CI 0.83 to 0.86$)^{[6]}$.

Schwatz SM et al found that women with total homocysteine $>/=15.6 \mathrm{micro} \mathrm{mol} / \mathrm{L}$ were at approximately twice the risk of MI as compare to women of total homocysteine $</=10 \mathrm{micromol} / \mathrm{L}$. Compared with control subjects, case patients had higher mean hooocysteine concentrations $(13.4+/-5.2$ versus $11.1+/-4.4 \mathrm{micromol} / \mathrm{L}$, $\mathrm{P}=.0004)$ and lower mean folate concentrations $(12.4+/-$ 13.4 versus $16.1+/-12.2 \mathrm{nmol} / \mathrm{L}, \mathrm{P}=.018)$. There was no association with vitamin B12 concentration. These data support the hypothesis that elevated homocysteine levels and low plasma folate are risk factors for MI among young women. ${ }^{[7]}$.

Elevated serum homocysteine levels interact with several other known cardiovascular risk factors like hypertension and smoking might imply that homocysteine levels should be reduced in high risk individuals or the people with a raised homocysteine level should be careful about controlling conventional risk factors.

The serum homocysteine levels in our cases is $29.77+/$ 6.97 as compared to $11+/-1.96$ in control group hence difference between the two groups in our study was highly significant ( $\mathrm{p}$ value $=.0001$ ) suggesting that homocysteine is an independent predictor of Coronary artery disease (MI).(Fig 2)

Jun-Hyun yoo et al, reports the mean plasma level of homocysteine was higher in cases than in controls $(11.8 \pm 5.6$ versus $9.6 \pm 4.1 \mu \mathrm{mol} / \mathrm{L} ; P=0.002)$. The proportion of subjects with moderate hyperhomocysteinemia was significantly higher in cases than in controls $(16.7 \%$ versus $5.0 \% ; P=0.004)$. The plasma homocysteine levels of patients having vessels with 3 or 2 stenosed sites were significantly higher than those of patients having vessels with 1 stenosed site or normal vessels $(14.6 \pm 1.4,11.0 \pm 1.4$ versus $7.8 \pm 1.5,8.9 \pm 1.4 \mu \mathrm{mol} / \mathrm{L}$ respectively; $P<0.02) .{ }^{[8]}$.

Saikat Datta et al reported that incidence of CVA was more in the male population, with a male: femaleratio being 1.6:1. The mean age of presentation was 61.7 +7.8 years. Biochemical assessment revealed that total LDL was significantly raised in those with intracerebral haemorrhages, whereas total serum homocysteine levels were significantly higher in those with intracerebral infarcts. hyperhomocysteinemia seems to be an important risk factor for ischemic stroke, although the prognosis of stroke probably does not depend on total plasma homocysteine concentrations. Therefore, fasting homocysteine level should be assessed in patients with first episode of ischemic cerebrovascular accidents (CVA), and also in those who are at risk of developing ischaemic CVA. ${ }^{[9]}$

The serum homocysteine levels in our cases is $28.14+/-$ 7.88 as compared to $11.18+/-1.82$ in control group hence difference between the two groups in our study was highly significant ( $\mathrm{p}$ value $=.0001$ ) suggesting that homocysteine is an independent predictor of Cerebrovascular disease. (Fig 3) 
It is observed in various studies that homocysteine interacts with hypertension, smoking and hyperlipidemia which imply that homocysteine levels should be reduced in high risk individuals or the persons with a raised homocysteine level should be particularly careful about conventional risk factor.

\section{Conclusion}

Elevated serum level of homocysteine is an independent risk factor for coronary artery disease and cererovascular stroke. In patients known to have cardiovascular disease and stroke, higher homocysteine levels increase the risk for future events. Selective screening for primary and secondary prevention should be considered for high risk patients.

\section{Reference}

1. Pearson TA, Blair SN, Daniels SR et al. AHA guidelines for primary prevention of cardiovascular disease and stroke: 2002 Update: Circulation. 2002; 106 (3):388-91.
2. Braunwald-6th edition. Heart disease. A textbook of cardiovascular medicine.

3. Donald W.J. Homocysteine and vitamins in cardiovascular disease. Clinical Chemistry. 1998; 44(8): 1833-1843

4. Eikelboom JW et al. Homocysteine and cardiovascular disease. A critical review of the epidemiological evidence. Annals of Internal Medicine.131:363-375, 1999.

5. Qujeq D, Omran TS, Hosini L. Correlation between total homocysteine, low-density lipoprotein cholesterol and highdensity lipoprotein cholesterol in the serum of patients with myocardial infarction. Clin Biochem. 2001; 34(2)97-101.

6. A meta analysis. Homocysteine and risk of ischemic heart disease and stroke: JAMA 2002; 288:2015.

7. Schwartz SM, Siscovick DS, Malinow MR, Rosendaal FR. $\mathrm{MI}$ in young women in relation to total plasma homocysteine. Circulation 1997 July 15:96(2):412-7.

8. Jun-Hyun Y, Chin-Sang C, Soo-Sang K et al: Relation of plasma homocysteine to cerebral infarction and cerebral atherosclerosis: Stroke. 1998 Dec;29:2478-2483.

*Corresponding author:

Dr. Dr. Hiral Samir Shah, Address: Above Samir Hospital, Lakdipul, Main Road Dandiabazar, Vadodara. 390001. Gujarat. India

Phone: +919879540188

Email: hiralsamir07@yahoo.co.in

Financial or other Competing Interests: None. 